\title{
Hypothermal Effects on Expression of Regucalcin, A Calcium Binding Protein, in Livers of Seawater- and Fresh Water-Acclimated Milkfish, Chanos Chanos
}

\section{Chia-Hao Chang}

National Chung Hsing University

Tsung-Han Lee ( $\boldsymbol{\nabla}$ thlee@email.nchu.edu.tw)

National Chung Hsing University

\section{Research Article}

Keywords: Chanos chanos, regucalcin, liver, low-temperature, salinity

Posted Date: March 25th, 2021

DOI: https://doi.org/10.21203/rs.3.rs-339395/v1

License: (c) (i) This work is licensed under a Creative Commons Attribution 4.0 International License.

Read Full License 


\section{Abstract}

Regucalcin (RGN) is a calcium-binding protein mainly expressed in the liver. It functions in regulating activities of several calcium-dependent enzymes related to energy metabolism, antioxidant mechanisms, and apoptotic pathways. Previous proteomics analyses revealed downregulation of regucalcin in milkfish livers when acclimated to low temperature $\left(18^{\circ} \mathrm{C}\right)$ from normal temperature $\left(28^{\circ} \mathrm{C}\right)$. This study first identified the full-length sequence of milkfish regucalcin from livers with high similarity in the protein structure and calcium-binding function compared to the regucalcin of other animals. The mRNA and protein expression of regucalcin in livers of fresh water (FW)- and seawater (SW)-acclimated milkfish under hypothermal acclimation were further analyzed. In FW milkfish, upregulation of regucalcin was found in mRNA and protein levels from two and four days, respectively, to one week after transfer to $18^{\circ} \mathrm{C}$ for the two. However, in SW milkfish, upregulation of regucalcin occurred quickly and returned to the basal levels in one (mRNA expression) or two days (protein expression) up until one week after transfer. These results suggested potential roles of regucalcin in maintaining calcium homeostasis and its correlation to differential physiological responses in livers of milkfish when they were acclimated to FW and SW.

\section{Introduction}

Regucalcin was discovered in 1978 as a calcium $\left(\mathrm{Ca}^{2+}\right)$ binding protein in the liver of rats (Yamaguchi and Yamamoto, 1978) and is known as the senescence marker protein-30 (SMP30) that decreases expression with ageing in rat livers (Fujita et al., 1992). Being a calcium-binding protein without EF-hand motif, several functions of regucalcin have been reported in mammals including intracellular calcium homeostasis, modulation of $\mathrm{Ca}^{2+}$ - or $\mathrm{Ca}^{2+} /$ calmodulin-dependent enzymes, participation of the biosynthesis process of ascorbic acid, and transcriptional regulation for several hormones (e.g., insulin and estrogen) (Fujita et al., 1998; Kondo et al., 2006; Yamaguchi, 2011; Yamaguchi, 2013). Many vertebrates have the ability to synthesize ascorbic acid, however this does not include teleosts due to their loss of an important key enzyme, gluconolactone oxidase (Ching et al., 2015). As a calcium binding protein, regucalcin was found to have inhibitory effects on several $\mathrm{Ca}^{2+}$-dependent kinase and enzymes (i.e., cAMP phosphodiesterase, caspase-3, and nitric oxide synthase) which inhibited apoptosis under stress (Matsutama et al., 2004; Izumi and Yamaguchi, 2004; Yamaguchi and Kurota, 1997). Regucalcin knockout in mice has been reported to elevate oxidative stress, antioxidant dysfunction, and hepatocyte apoptosis, leading to liver fibrosis and even death (Park et al., 2010; Kondo et al., 2006). Therefore, regucalcin has critical functions in calcium homeostasis for several physiological responses. Related studies on teleosts, however, are limited.

Hypothermal stress was found to change cell membrane fluidity and protein structure that may lead to rearrangement of cytoskeleton, activation of calcium channels, or ER stress-induced imbalance of cytosolic $\mathrm{Ca}^{2+}$ levels, which then affect several physiological responses (Bayley et al., 2018; Wang et al., 2019). Meanwhile, hypothermal stress may induce reactive oxygen species (ROS) due to the immune 
response or mitochondria dysfunction (Donaldson et al., 2008). Elevation of cytoplasmic $\mathrm{Ca}^{2+}$ levels was found in pufferfish upon low-temperature challenge, leading to oxidative stress and apoptosis (Cheng et al., 2018). Several calcium binding proteins were reported to be involved in mechanisms of cold tolerance in fish. The livers of the Antarctic notothenioid fish (Dissostichus mawsoni; Dm) contained very high levels of calmodulin. Overexpression of $D m$-calmodulin further demonstrated increased cold tolerance in tobacco (Na et al., 2013). In addition, feed supplemented with Dm-calmodulin recombinant protein has enhanced cold tolerance in juvenile, orange-spotted grouper (Epinephelus coioides), showing increased antioxidant enzyme activity and reduced oxidative stress responses upon low-temperature challenge (Luo et al., 2015). In the fruit fly, high expression of the Drosophila-cold-acclimation (Dca) gene, a regucalcinlike protein with the function of maintaining calcium homeostasis, was found to help the fly tolerate cold treatments (Arboleda-Bustos and Segarra, 2011). A proteomic analysis revealed that the protein spot corresponding to regucalcin in seawater (SW) milkfish livers disappeared after one-week acclimation to $18^{\circ} \mathrm{C}$ (Chang et al., 2016b). Hence, it is suggested that regucalcin may play roles in maintaining calcium homeostasis in milkfish. Furthermore, the disappearance of the regucalcin protein spot in livers of hypothermal milkfish suggested functional deterioration of regucalcin, together with elevation of oxidative stress and dysfunction of antioxidant mechanisms (Chang et al., 2016b). In zebrafish, regucalcin was found to express mainly in the liver, corresponding to liver injury. Meanwhile, mRNA expression of zebrafish regucalcin (rgn) decreased with aging (Fujisawa et al., 2011). In rainbow trout, the TCO and BORN strains revealed different patterns of rgn expression in several tissues including the liver, head kidney, and muscle. After 21-days infection with Aeromonas salmonicida, the rgn was up-regulated in the liver of only the TCO strain. In addition, lower and higher temperature challenges revealed significant differences in expression of rgn between the TCO and BORN strain. There was no comparison, however, in the liver, head kidney, trunk kidney, and muscle between different temperature groups (Verleih et al., 2011). Although plasma calcium in milkfish was not changed under hypothermal challenge, calcium imbalance may happen on cellular levels according to the proteomics analysis (Kang et al., 2015; Chang et al., 2016b). Therefore, regucalcin could be considered a novel indicator of milkfish for hypothermal acclimation.

Milkfish is a tropical species with high mortality during cold snaps in winter in Southeast Asia (Fachry et al., 2018; Liao, 1991; Martinez et al., 2006). Being a euryhaline aquaculture species, milkfish have the ability to survive in environments with a broad range of salinities and thus have been cultured in water with different salinities (Jana et al., 2006). In previous studies, SW-acclimated milkfish showed better lowtemperature tolerance than fresh water (FW)-acclimated milkfish (Kang et al., 2015). When acclimated to low-temperature environments, different strategies in energy metabolism and antioxidant mechanisms were found in the livers of FW- and SW-acclimated milkfish (Chang et al., 2016a, 2017, 2018, 2019). Since regucalcin, a calcium binding protein, has regulatory functions for energy metabolism, antioxidant mechanisms, and apoptosis in mammals (Fukaya and Yamaguchi, 2004; Yamaguchi, 2013; Vaz et al., 2016), the regucalcin in the liver of milkfish was thought to be an upstream regulator for differential physiological responses under salinity and hypothermal acclimation. Hence, this study reported the molecular characteristics, gene expression, and relative protein abundance of regucalcin in livers of FW- 
and SW-acclimated milkfish to illustrate a potential mechanism of modulating cellular calcium for hypothermal acclimation.

\section{Materials And Methods}

\subsection{Fish and experimental conditions}

Juvenile milkfish (Chanos chanos, total length: 9-10 cm, weight range: 10-12 g) were obtained from a local fish farm in Changhua, Taiwan. Fish were acclimated and maintained in $400 \mathrm{~L}$ tanks containing FW and SW (35\%o). Milkfish were maintained in a $12 \mathrm{~h}$ light/12 $\mathrm{h}$ dark photoperiod at $28.0 \pm 0.5^{\circ} \mathrm{C}$. The experimental SW was prepared from local tap water and Blue Treasure Sea Salts (New South Wales, Australia). The water was continuously recirculated through fabric-floss filters and partially replaced every month. The fish were fed daily with commercial pellets (Fwusow Industry, Taichung, Taiwan). The experimental procedures were approved by the Institutional Animal Care and Use Committee (IACUC) of the National Chung Hsing University, Taichung, Taiwan (IACUC Approval No. 105 - 024 to THL).

The cooling system was comprised of two $100 \mathrm{~L}$ tanks; one $100 \mathrm{~L}$ filter tank, and one tank fitted with an A/C compressor (PF-225M, Prince, Tainan, Taiwan) that had an electronic temperature controller. The temperature of the hypothermal treatment as $18^{\circ} \mathrm{C}$ was determined according to our previous study on the non-lethal cold tolerance temperature of milkfish (Chang et al., 2016b). Milkfish were transferred to the cooling tanks with FW or SW for at least two days prior to beginning the hypothermal experiments to reduce the handling stress. Then the water temperatures in the cooling tanks were lowered at a constant rate $\left(2^{\circ} \mathrm{C} \mathrm{h}^{-1}\right)$ from 28 to $18^{\circ} \mathrm{C}$. Milkfish were kept at $18^{\circ} \mathrm{C}$ for one week before sampling. For the hypothermal time-course experiments, the milkfish were sampled after hypothermal treatment $\left(18^{\circ} \mathrm{C}\right)$ for $1,3,6,12,24,48,96$, and $168 \mathrm{~h}$. All experimental fish were anesthetized with $0.5 \%$ 2-phenoxyethanol before sampling. The livers of milkfish were dissected quickly, immersed in liquid nitrogen, and stored at $-80^{\circ} \mathrm{C}$ for the following analyses.

\subsection{Total RNA extraction and cDNA preparation}

The method used for RNA isolation was modified by Chang et al. (2016a). The RNA samples were extracted using a TRI reagent (Molecular Research Center, Cincinnati, OH, USA). All samples were homogenized using a Pellet Pestle® Cordless Motor (Kimble Chase, Millvile, NJ, USA). The quality of the extracted total RNA was determined by the NanoDrop 2000 (Thermo, Wilmington, CA, USA) and was evaluated by $1.0 \%$ agarose gel electrophoresis. $1 \mu \mathrm{g}$ of total RNA was used for CDNA synthesis using iScript ${ }^{\text {TM }}$ Reverse Transcription Supermix (Bio-Rad, Hercules, CA, USA) according to the manufacturer's protocol. The templates of cDNA for full-length sequence cloning were obtained using the SMART RACE cDNA amplification kit (Clontech, Palo Alto, CA, USA) following manufacturer's instructions.

\subsection{Sequence cloning, characterization and analysis}

Primers were designed by Primer3Plus software (http://www.bioinformatics.nl/cgibin/primer3plus/primer3plus.cgi) based on a highly conserved region compared with several sequences 
of teleosts. The PCR products were subcloned into the pGM-T vector (GeneMarek, Taipei, Taiwan) and sequencing was conducted. The full-length sequence was obtained following RACE manuscript's instructions. All primers are listed in Table S1. The open reading frame of Ccrgn was predicted by ORF finder (https://www.ncbi.nlm.nih.gov/orffinder/). The sequence prediction analyses were obtained with DTU Bioinformatics (http://www.cbs.dtu.dk/services/), ExPASy (https://www.expasy.org/), Uniport (https://www.uniprot.org/), and SWISS-MODEL (https://swissmodel.expasy.org/). The phylogenetic tree was constructed using Mega-X using the Maximum likelihood method with 1,000 bootstraps.

\subsection{Real-time PCR}

The qPCR was performed on a MiniOpicon real-time PCR (Bio-Rad). The qPCR mixture contained $8 \mu \mathrm{L}$ of cDNA (100-fold dilution), $2 \mu \mathrm{L}$ of $2 \mu \mathrm{M}$ primer pairs, and $10 \mu \mathrm{L}$ of $2 X$ KAPA SYBR $\circledast$ FAST qPCR Master Mix (Kapa Biosystems, Wilmington, MA, USA). The qPCR process was conducted using the following protocol: activation step $\left(95^{\circ} \mathrm{C}\right)$ for $10 \mathrm{~min}, 40$ cycles of $10 \mathrm{~s}$ denaturation step $\left(95^{\circ} \mathrm{C}\right)$, an annealing/extension step $\left(60^{\circ} \mathrm{C}\right)$ for $30 \mathrm{~s}$, and a melting curve analysis from $70^{\circ} \mathrm{C}$ to $95^{\circ} \mathrm{C}$. The $\mathrm{qPCR}$ primers were qualified by amplification efficiency, melting curve, single product in electrophoresis, and sequencing. Glyceraldehyde 3-phosphate dehydrogenase ( $g a p d h$ ) was analyzed as the normalization gene in the present experiment. The relative expression was obtained using the formula $2^{-[(\mathrm{Ct} r g n, \mathrm{n}-\mathrm{Ct}}$ gapdh, n) - (Ct rgn, c - Ct gapdh, c)], where "Ct" was the threshold cycle number, " $n$ " indicated each sample, and "c" indicated the control mixed with cDNA samples of all experiments.

\subsection{Western blot analysis}

For immunoblotting analysis, the milkfish liver was placed into a $2.0 \mathrm{~mL}$ microcentrifuge tube and a SEID buffer was added (150 mM sucrose, $10 \mathrm{mM}$ EDTA, $50 \mathrm{mM}$ imidazole, $0.1 \%$ sodium deoxycholate, $\mathrm{pH} 7.5$ ) containing a protease inhibitor (vol/vol: 25:1; Roche, Mannheim, Germany). Then the sample in the tube was homogenized using a Polytron PT1200E homogenizer (Lucerne, Switzerland) on ice. The supernatant concentration of the homogenate was determined using the Pierce ${ }^{\mathrm{TM}} \mathrm{BCA}$ protein assay kit (Thermo Fisher Scientific, Waltham, MA, USA). All samples were heated at $60^{\circ} \mathrm{C}$ for 15 min with a $6 \mathrm{X}$ protein sample buffer $(0.5 \mathrm{M}$ Tris- $\mathrm{Cl}, 40 \%$ glycerol, $10 \%$ sodium dodecyl sulfate, $9.3 \%$ DTT, $0.0012 \%$ bromphenol blue, $\mathrm{pH}$ 6.8). $100 \mu \mathrm{g}$ denatured protein samples were fractionated by $10 \%$ polyacrylamide gel. Gels were electrophoresed and transferred to $0.45 \mu \mathrm{m}$ PVDF membranes (Millipore, Bedford, MA, USA). Membranes were blocked with $5 \%(\mathrm{w} / \mathrm{v})$ nonfat dried milk in PBST (phosphate-buffered saline with $0.1 \%$ Tween-20) for $1 \mathrm{~h}$. PVDF membranes were subsequently incubated with the SMP30 antibody (sc377184, Santa Cruz Biotech., Dallas, TX, USA) or GAPDH antibody (GTX100118; GeneTex, Irvine, CA, USA) overnight at $4^{\circ} \mathrm{C}$. Regucalcin $\left(0.2 \mu \mathrm{g} \mathrm{mL}^{-1}\right)$ and GAPDH $\left(0.1 \mu \mathrm{g} \mathrm{mL}^{-1}\right)$ antibodies were diluted with $1 \%$ bovine serum albumin (CyrusBioscience, Taipei, Taiwan) in PBST. The secondary antibody (goat antirabbit IgG antibody HRP, GTX213110-01, GeneTex; rabbit anit-mouse IgG antibody HRP, GTX213112-01, GeneTex) was incubated at room temperature for $1 \mathrm{~h}$. The western chemiluminescence HRP substrate (TPro Biotechnology, Taipei, Taiwan) was used to develop the immunoblots. Images were photographed with a cool-charge-coupled device (CCD) camera (ChemiDoc XRS ${ }^{+}$, Bio-Rad) and analyzed using Image Lab v. 3.0 software (Bio-Rad). The intensity of immunoreactive bands was calculated to numerical values 
and normalized against GAPDH. The PVDF membrane was reused one-time for GAPDH antibody loading control, by added striping buffer ( $63 \mathrm{mM}$ Tris- $\mathrm{HCl}, 70 \mathrm{mM}$ sodium dodecyl sulfate, $\mathrm{pH} 6.8$ ) with $0.7 \% \beta$ mercaptoethanol (Millipore) at $50^{\circ} \mathrm{C}$ for $30 \mathrm{~min}$ and re-blocked following the method described above.

\subsection{Chromatin immunoprecipitation (ChIP)}

Chromatin immunoprecipitation (ChIP) was performed using an EpiQuik ${ }^{\text {TM }}$ Tissue Chromatin Immunoprecipitation Kit (Epigentek, Farmingdale, NY, USA). 40 mg milkfish livers were cross-linked with $1 \%$ formaldehyde, and unreacted formaldehyde was quenched by $125 \mathrm{mM}$ glycine. The liver was then homogenized in homogenizing buffer (Epigentek) using a plastic rod in the $1.5 \mathrm{~mL}$ microcentrifuge tube with 10-20 strokes. After centrifugation $\left(5000 \mathrm{~g}, 5 \mathrm{~min}, 4^{\circ} \mathrm{C}\right)$, the pellets were suspended in the nuclear lysis buffer (Epigentek) and washed by phosphate-buffered saline (PBS). The cell lysis was conducted with 5 pulses of $20 \mathrm{sec}$ using a sonicator (Branson, Brookfield, CT, USA), and the resulting DNA was found to be between 200-1,000 bp. The recoating-strip was incubated with a polyclonal antibody against antiSMP30 (sc377184, Santa Cruz) or IgG (Epigentek) as the negative control at room temperature for 90 min. The coated-strip was washed twice with the antibody-diluted buffer (Branson). The protein-DNA complexes were reverse crosslinked using Proteinase $\mathrm{K}$ incubated at $65^{\circ} \mathrm{C}$ for $15 \mathrm{~min}$. The extracted DNA was purified using spin columns for cleanup. For qPCR analysis, immunoprecipitated DNA and input DNA (un-immunoprecipitated) were used as templates. The qPCR primers for ChIP-qPCR are listed in Table S1. Subsequent qPCR analysis followed the previously explained methods.

\subsection{Statistical analysis}

Values were expressed as means \pm SEM (standard error of the mean). Data on tissue distribution and hypothermal time-course experiments were compared using a one-way ANOVA with Tukey's and Dunnet's test, respectively. Data from the hypothermal experiments were compared with a two-way ANOVA with Tukey's HSD post-hoc methods. All experimental data analysis was conducted using R software ( $R$ Foundation, Vienna, Austria). Significant differences were accepted when $P<0.05$.

\section{Results}

\subsection{Identification and characterization of regucalcin in milkfish}

According to the transcriptomic library of milkfish and PCR cloning, the regucalcin gene was identified in milkfish (Fig. 1A). The full-length cDNA of regucalcin (MT218432) contained 1,603 bp including an ORF of $891 \mathrm{bp}$, a 5'-UTR and 3'-UTR of $79 \mathrm{bp}$ and $821 \mathrm{bp}$, respectively. The ORF encoded 296 amino acids with a molecular weight of $33 \mathrm{kDa}$ and theoretical isoelectric point (pl) of 5.59. The sequence was predicted to have one phosphorylation site at S14 and no signal peptide. The predicted protein structure of regucalcin with calcium dock-site (green spot) exhibited the calcium binding residues (E18, N103, N151, and D201; Fig. 1B) and the gluconolactonase (GNL) [EC 3.1.1.17] region $\left(\mathrm{V}^{9}-\mathrm{S}^{261}\right)$. 


\subsection{Phylogenetic tree analysis and tissue distribution of regucalcin expression}

The phylogenetic analyses revealed that milkfish regucalcin (RGN) was highly similar in the branch of Ostariophysi including channel catfish (Ictalurus punctatus, 85.6\%), goldfish (Carassius auratus; 83.5\%), and zebrafish (Danio rerio; 86.1\%). According to different branches of subclasses the similarities ranged from 71 to $82 \%$ compared to other teleosts (Fig. 1C). The expression of regucalcin gene (Ccrgn) was detectable in all analyzed tissues. The highest expression of Ccrgn was found in the liver followed by the intestine (Fig. 2).

\subsection{Effects of hypothermal treatments on hepatic regucalcin expression in SW- and FW-acclimated milkfish}

Under one-week hypothermal acclimation, the hepatic rgn was up-regulated in FW-acclimated milkfish and down-regulated in SW-acclimated milkfish. The Ccrgn expression in livers of the FW/ $18^{\circ} \mathrm{C}$ group was significantly higher than the SW/18 ${ }^{\circ} \mathrm{C}$ group (Fig. 3). Two-way ANOVA analyses revealed that $\mathrm{Ccrgn}$ expression was affected by the hypothermal treatment $\left(\mathrm{F}_{1,23}=9.92, p=0.01\right)$ and environmental salinity $\left(F_{1,23}=20.12, p=0.01\right)$. Synergistic interaction effects of hypothermal stress and salinity significantly affected the expression of Ccrgn (Fig. 3). Upon hypothermal challenge, hepatic Ccrgn expression was significantly up-regulated at $1 \mathrm{~h}$ and from $48 \mathrm{~h}$ to one week after transfer to $18^{\circ} \mathrm{C}$ in the $\mathrm{FW}$ group (Fig. 4A). On the other hand, Ccrgn expression was found to increase significantly at 3, 6, and $12 \mathrm{~h}$ and return to normal level from $24 \mathrm{~h}$ to one-week post transfer in livers of SW-acclimated milkfish (Fig. 4B).

\subsection{The protein profiles of hepatic regucalcin under hypothermal acclimation}

The single immunoreactive band of regucalcin in the milkfish liver was detected at $35 \mathrm{kDa}$, and GAPDH was used as the loading control for normalization. Under hypothermal acclimation, the protein abundance of regucalcin in FW-acclimated milkfish livers was upregulated, but in SW-acclimated milkfish there was no significant difference. In addition, relative abundance of RGN in livers of the $F W / 18^{\circ} \mathrm{C}$ group was significantly higher than that of the $S W / 18^{\circ} \mathrm{C}$ group (Fig. 5). Two-way ANOVA analyses revealed synergistic interaction effects $\left(F_{1,23}=23.25, p=0.03\right)$ between the hypothermal treatment and ambient salinities on protein abundance of RGN. The factors of the low temperature $\left(F_{1,23}=13.05, p=0.23\right)$ and environmental salinities $\left(\mathrm{F}_{1,23}=3.25, p=0.15\right)$, however, were not significantly different between $\mathrm{FW}$ - and SW-acclimated milkfish (Fig. 5). Upon hypothermal challenge, RGN protein abundance of FW milkfish livers increased gradually from $12 \mathrm{~h}$ after transfer to $18^{\circ} \mathrm{C}$ and became significantly elevated from $96 \mathrm{~h}$ at $18^{\circ} \mathrm{C}$ (Fig. 6A). In the SW groups, relative amounts of hepatic RGN were increased from $1 \mathrm{~h}$ after transfer to $18^{\circ} \mathrm{C}$, became significantly up-regulated at 3 and $24 \mathrm{~h}$, and returned to normal level after $48 \mathrm{~h}$ to one week at $18^{\circ} \mathrm{C}$ (Fig. 6B).

\section{Discussion}


The full-length regucalcin sequence of the milkfish was cloned in the present study. The predicted protein structure was highly similar (76.3\%) to human regucalcin with multiple functions including GNL for use in vitamin $\mathrm{C}$ biosynthesis pathway and calcium chelator for use in calcium homeostasis (Chakraborti and Bahnson, 2011). However, teleosts cannot synthesize vitamin $C$ due to the lack of a required enzyme, glulonolactone oxidase, in the biosynthesis pathway (Linster and Schaftingen, 2007; Ching et al., 2015). In addition, no genetic information regarding glulonolacton oxidase can be found in the genome database for milkfish (http://140.120.209.83/CCD/index/C.chanos_home.htm). Regucalcin in teleosts should play major roles in maintaining calcium homeostasis and modulating calcium-dependent physiological responses. The residues, E18, N103, N151, and D201 in milkfish regucalcin sequence were highly conservative compared to the calcium binding residues of human regucalcin (Kondo et al., 2006; Chakraborti and Bahnson, 2011). The amino acid sequence of milkfish regucalcin was also highly similar (72-86\%) to that of the other teleosts with conserved calcium binding residues.

To date, there are few studies on teleostean regucalcin. The present study revealed that Ccrgn was mainly distributed in the liver. In zebrafish, regucalcin was also mainly expressed in the liver (Fujisawa et al., 2011). In rainbow trout, however, regucalcin was found mainly in the liver and kidneys (Verleih et al., 2011). Expression levels of regucalcin in zebrafish decreased with aging which might be due to the deteriorated ability of maintaining calcium homeostasis (Fujisawa et al., 2011). A previous proteomics study on livers of SW-acclimated milkfish identified regucalcin and found the corresponding protein spot disappeared in two-dimensional gel (2D-gel) electrophoresis under hypothermal acclimation (Chang et al., 2016b). The immunoblots in the present study, however, showed that relative abundance of regucalcin in livers of the $S W / 18^{\circ} \mathrm{C}$ group did not decrease significantly compared to the $S W / 28^{\circ} \mathrm{C}$ group. A change in the isoelectric point of the protein via phosphorylation or glycosylation might be the reason that the corresponding protein spot of regucalcin disappeared in 2D-gel electrophoresis but relative protein abundance of regucalcin remained high in livers of SW-acclimated milkfish under hypothermal acclimation.

$\mathrm{Ca}^{2+}$ is a common second messenger for cells (Das et al., 2018). In mammals, the calcium signal pathway is highly correlated to cold tolerance ability and affects several physiological responses including anti-apoptosis, antioxidant capacity, and transcriptional regulator mechanisms (Kondo et al., 2006; Yamaguchi, 2011, 2013). In pufferfish, the concentration of free $\mathrm{Ca}^{2+}$ in the cytoplasm was upregulated at lower temperatures, leading to apoptotic responses (Cheng et al., 2018). Regucalcin was reported to play an important role in regulating cytoplasmic free-Ca ${ }^{2+}$ levels and inhibiting excess $\mathrm{Ca}^{2+}$ influx to prevent apoptosis and suppress glycolytic metabolism in rats (Fujita et al., 1998; Vaz et al., 2016). Therefore, regucalcin may play similar roles in modulating $\mathrm{Ca}^{2+}$ in teleosts. Under hypothermal acclimation, the proteomics analysis revealed upregulation of regucalcin protein in the hepatopancreas of the white shrimp, Litopenaeus vannamei (Fan et al., 2016). The homologue of regucalcin in Drosophila was found to be upregulated during cold acclimation and was suggested to play an important role in cold tolerance ability (Goto, 2000; MacMillan et al., 2016). On the other hand, calmodulin, the other calcium binding protein, showed very high levels in livers of the Antarctic fish (D. mawsoni). Overexpression of 
calmodulin in tobacco enhanced cold tolerance ability of the Antarctic fish (Na et al., 2013). In this study, milkfish raised in FW and SW showed differential profiles of regucalcin expression in both mRNA and protein levels upon hypothermal challenge. In FW-acclimated milkfish, the mRNA level was up-regulated after two days and protein levels became significantly higher from four days to one week after transfer to $18^{\circ} \mathrm{C}$. In SW-acclimated milkfish, mRNA expression was up-regulated and returned to the basal level in 1 day and for protein expression it was up-regulated in two days and until seven days after the transfer to $18^{\circ} \mathrm{C}$. Unlike the $\mathrm{FW}$-acclimated milkfish, the regucalcin-involved physiological responses in SWacclimated milkfish livers seemed to be consistent under hypothermal acclimation and the expression levels returned in one day at low temperatures. Kang et al. (2015) reported that SW-acclimated milkfish have a better cold tolerant ability than FW-acclimated individuals. Although plasma calcium concentration in FW-acclimated milkfish was not changed during hypothermal acclimation (Kang et al., 2015), cellular calcium concentration in livers of FW-acclimated milkfish might be imbalanced upon acute hypothermal challenge (Kang et al., 2015), like those found in the pufferfish (Cheng et al., 2018). Imbalanced calcium contents might be one of the reasons of induced apoptosis of hepatocytes in milkfish with low-temperature treatments (Izumi and Yamaguchi, 2004; Yamaguchi, 2013; Chang et al., 2018; Cheng et al., 2018; Chang et al., 2020). In addition, it is possible that in milkfish livers the fluctuation in calcium contents led to degradation of hepatic glycogen to provide energy for maintaining normal physiological responses, as was found in mice (Oliva-Vilarnau et al., 2018). On the other hand, the same patterns of regucalcin expression in transcriptional and translational levels in livers of SWacclimated milkfish upon hypothermal challenge suggested that SW milkfish have the ability to maintain cellular calcium homeostasis under hypothermal acclimation.

In previous mammalian studies, regulation of hepatic regucalcin expression was demonstrated by administration of calcium ions. The fluctuation in calcium levels affected MAPK kinase, protein kinase $C$, and calmodulin kinase, as well as activated binding of transcription factors (AP-1, NF1-A1, and RGPR-

p117) to specific binding sites in the sequence of regucalcin to modulate its gene expression (Murata and Yamaguchi, 1998; Yamaguchi, 2011). In addition, regucalcin is suggested to be a transcription factor for regulating expression of downstream genes in mice (Yamaguchi, 2013). Since the sequence of Ccrgn regucalcin was predicted to contain several binding sites of transcription factors (i.e., AP-1, $\beta$-catenin, C/EBP) as well as the calcium binding sites (i.e., E18, N103, N151, and D201), the transcription activities of different calcium signaling pathways were supposed to be modulated by regucalcin in milkfish as in mammals.

\section{Conclusions}

In this study, full-length regucalcin in milkfish was identified and suggested to play roles for maintaining homeostasis of cellular calcium. SW-acclimated milkfish has better cold tolerance ability than FWacclimated individuals (Kang et al., 2015). The regucalcin expression found in this study suggested that the ability for maintaining calcium homeostasis in livers was also better in SW-acclimated milkfish rather than FW ones under an acute phase of hypothermal acclimation. The molecular evidence in this study 
further illustrates that salinity is an important factor that affects the maintenance of homeostasis in the euryhaline milkfish under hypothermal challenge.

\section{Declarations}

\section{Acknowledgements}

This work was partly supported by a grant to T.H. Lee from the Ministry of Science and Technology (MOST) of Taiwan (105-2313-B-005-027-MY3 and 108-2313-B-005-006-MY3) and in part by the integrative Evolutionary Galliform Genomics (iEGG) and Animal Biotechnology Center from The Feature Area Research Center Program within the framework of the Higher Education Sprout Project by the Ministry of Education (MOE) in Taiwan (109-S-0023-F). C.H.C. was supported by the postdoctoral fellowships from the MOST (108-2811-B-005-520).

\section{Author Declarations}

\section{Funding}

This work was partly supported by a grant to T.H. Lee from the Ministry of Science and Technology (MOST) of Taiwan (105-2313-B-005-027-MY3 and 108-2313-B-005-006-MY3) and in part by the integrative Evolutionary Galliform Genomics (iEGG) and Animal Biotechnology Center from The Feature Area Research Center Program within the framework of the Higher Education Sprout Project by the Ministry of Education (MOE) in Taiwan (109-S-0023-F). C.H.C. was supported by the postdoctoral fellowships from the MOST (108-2811-B-005-520).

\section{Conflicts of interest/Competing interests}

No conflicts of interest are declared by the authors.

\section{Ethics approval/declarations}

The experimental procedures were approved by the Institutional Animal Care and Use Committee (IACUC) of the National Chung Hsing University, Taichung, Taiwan (IACUC Approval No. 105-024 to THL).

\section{Consent to participate}

Not applicable

\section{Consent for publication}

All authors read and approved the final manuscript for publication.

\section{Availability of data and material/Data availability}

Not applicable 


\section{Code availability}

Not applicable

\section{Authors' contributions}

C.H.C conceived and designed this study and contributed to manuscript writing. T.H.L. reviewed and edited the manuscript. T.H.L. supervised the project. All authors read and approved the final manuscript for publication.

\section{Disclosures}

No conflicts of interest are declared by the authors.

\section{References}

1. Arboleda-Bustos, C.E., Segarra, C., 2011. The Dca gene involved in cold adaptation in Drosophila melanogaster arose by duplication of the ancestral regucalcin gene.

2. Bayley, J.S., Winther, C.B., Andersen, M.K., Grønkjær, C., Nielsen, O.B., Pedersen, T.H., Overgaard, J., 2018. Cold exposure causes cell death be depolarization mediated $\mathrm{Ca}^{2+}$ overload in a chillsusceptible insect. Proc. Natl. Acad. Sci. USA 115, E9737-E9744.

3. Chakraborti, S., Bahnson, B.J., 2011. Crystal structure of human senescence marker protein 30; insights linking structural enzymatic and physiological functions. Biochemistry 49, 3436-3444.

4. Chang, C.H., Huang, J.J., Yeh, C.Y., Tang, C.H., Hwang, L.Y., Lee, T.H., 2018. Salinity effects on strategies of glycogen utilization in liver of euryhaline milkfish (Chanos chanos) under hypothermal stress. Front. Physiol. 10, 81.

5. Chang, C.H., Liu, Z.Z., Lee, T.H., 2019. Changes in hypothermal stress induced hepatic mitochondrial metabolic patterns between fresh water- and seawater-acclimated milkfish, Chanos chanos. Sci. Rep. $9,18502$.

6. Chang, C.H., Lo, W.Y., Lee, T.H., 2016a. The antioxidant peroxiredoxin 6 (Prdx6) exhibits different profiles in the livers of seawater- and fresh water-acclimated milkfish, Chanos chanos, upon hypothermal challenge. Front. Physiol. 7, 580.

7. Chang, C.H., Tang, C.H., Kang, C.K., Lo, W.Y., Lee, T.H., 2016b. Comparison of integrated responses to nonlethal and lethal hypothermal stress in milkfish (Chanos chanos): a proteomics study. PLoS One 11, e0163538.

8. Cheng, C.H., Guo, Z.X., Wang, A.L., 2018. The protective effects of taurine on oxidative stress, cytoplasmic free- $\mathrm{Ca}^{2+}$ and apoptosis of pufferfish (Takifugu obscurus) under low temperature. Fish Shellfish Immun. 77, 457-464.

9. Ching, B., Chew, S.F., Ip, Y.K., 2015. Ascorbate synthesis in fishes: a review. IUBMB Life 67, 69-76. 
10. Das, C., Thraya, M., Vijayan, M.M., 2018. Nongenomic cortisol signaling in fish. Gen. Comp. Endocrinol. 265, 121-127.

11. Donaldson, M.R., Cooke, S.J., Patterson, D.A., Macdonald, J.S., 2008. Cold shock and fish. J. Fish Biol. 73, 1491-1530.

12. Dos Santos, R.S., Galina, A., Da-Silva, W.S., 2013. Cold acclimation increases mitochondrial oxidative capacity without inducing mitochondrial uncoupling in goldfish white skeletal muscle. Biol. Open 2 , 82-87.

13. Fachry, M. E., Sugama, K., Rimmer, M. A., 2018. The role of small-holder seed supply in commercial mariculture in South-east Asia. Aquac. 495, 912-918.

14. Fujisawa, K., Terai, S., Hirose, Y., Takami, T., Yamamoto, N., Sakaida, I., 2011. Senescence marker protein 30 (SMP30)/regulcacin (RGN) expression decreases with aging, acute liver injuries and tumors in zebrafish. Biochem. Biophys. Res. Commun. 414, 331-336.

15. Fujita, T., Shirasawa, T., Inoue, H., Kitamura, T., Marutama, N., 1998. Hepatic and renal expression of senescence marker protein-30 and its biological significance. J. Gastroenterol. Hepatol. 13, S124.S131.

16. Fujita, T., Uchida, K., Maruyama, N., 1992. Purification of senescence marker protein-30 (SMP30) and its androgen-independent decrease with age in the rat liver. Biochim. Biophys. Acta 1116, 122-128.

17. Fukaya, Y., Yamaguchi, M., 2004. Regucalcin increases superoxide dismutase activity in rat liver cytosol. Biol. Pharm. Bull. 27, 1444-1446.

18. Goto, S.G., 2000. Expression of Drosophila homologue of senescence marker protein-30 during cold acclimation. J Insect Physiol. 46, 1111-1120.

19. Izumi, T., Yamaguchi, M., 2004. Overexpression of regucalcin suppresses cell death and apoptosis in cloned rat hepatoma H4-II-E cells induces by lipopolysaccharide, PD 98059, dibucaine, or Bay K 9644. J. Cell Biochem. 15, 598-608.

20. Jana, S.N., Garg, S.K., Patra, B.C., 2006. Effect of inland water salinity on growth performance and nutritional physiology in growing milkfish, Chanos chanos (Forsskal): field and laboratory studies. J. Appl. Ichthyol. 22, 25-34.

21. Kang, C.K., Chen, Y.C., Chang, C.H., Tsai, S.C., Lee, T.H., 2015. Seawater-acclimation abates cold effects on $\mathrm{Na}^{+}, \mathrm{K}^{+}$-ATPase activity in gills of the juvenile milkfish, Chanos chanos. Aquaculture, 446, 67-73.

22. Kondo, Y., Inai, Y., Sato, Y., Handa, S., Kubo, S., Shimodado, K., Goto, S., Nishikimi, M., Maruyama, N., Ishigami, A., 2006. Senescence marker protein 30 functions as gluconolactonase in L-ascorbic acid biosynthesis and its knockout mice are prone to scurvy. Proc. Natl. Acad. Sci. U.S.A. 103, 5723-5728.

23. Liao, I. C., 1991. Milkfish culture in Taiwan. In: McVey, J. P. (Ed.), CRC Handbook of Mariculture Volume II: Finfish Auaculture. CRC Press, Boca Raton, Florida, pp. 91-115.

24. Linster, C.L., Schaftingen, E.V., 2007. Vitamin C biosynthesis, recycling and degradation in mammals. FEBS J. 274, 1-22. 
25. Luo, S.W., Wnag, W.N., Cai, L., Qi, Z.H., Wang, C., Liu, Y., Peng, C.L., Chen, L.B., 2015. Effects of a Dissostichus mawsoni-CaM recombinant proteins feed additive on juvenile orange-spotted grouper (Epinephelus coioides) under the acute low temperature challenge. Fish Physiol. Biochem. 41, 13451358.

26. Martinez, F. S., Tseng, M. C., Yeh, S. P., 2006. Milkfish (Chanos chanos) culture: situations and trends. J. Fish. Soc. Taiwan 33, 229-244.

27. Matsutama, S., Kitamura, T., Enomoto, N., Fujita, T., Ishigami, A., Handa, S., Marutama, N., Zheng, D., Ikejima, K., Takei, Y., Sato, N., 2004. Senescence marker protein-30 regulates Akt activity and contributes to cell survival in HepG2 cells. Biochem. Bioph. Res. Co. 321, 386-390.

28. Murata, T., Yamahuchi, M., 1998. $\mathrm{Ca}^{2+}$ administration stimulates the binding of AP-1 factor to the $5^{\prime}-$ flanking region of the rat gene for the Ca2+-binding protein regucalcin. Biochem. J. 329, 157-163.

29. Na, Y., Changlian, P., Deng, C., Qiao, H., Guanghui, X., Fei, G., Liangbiao, C., 2013. The over-expression of calmodulin from Antarctic notothenioid fish increases cold tolerance in tobacco. Gene 521, 32-37.

30. Nejak-Bowen, K.N., Zeng, G., Tang, X., Cieply, B., Monga, S.P., 2009. $\beta$-catenin regulates vitamin C biosynthesis and cell survival in murine liver. J. Biol. Chem. 284, 28115-28127.

31. Oliva-Vilarnau, N., Hankeova, S., Vorrink, S.U., Mkrtchian, S., Andersson, E.R., Lauschke, V.M., 2018. Calcium signaling in liver injury and regeneration. Front. Med. 5, 192.

32. Park, J.K., Ki, M.R., Lee, H.R., Hong, I.H., Ji,A.R., Ishigami, A., Park, S.I., Kim, J.M., Chung, H.Y., Yoo, S.E., Jeong, K.S., 2010. Vitamin $C$ deficiency attenuates liver fibrosis by way of up-regulated peroxisome proliferator-activated receptor-gamma expression in senescence marker protein 30 knockout mice. Hepatology 51, 1766-1777.

33. Vaz, C.A., Marques, R., Cardoso, H.J., Maia, C.J., Socorro, S., 2016. Suppressed glycolytic metabolism in the prostate of transgenic rats overexpressing calcium-binding protein regucalcin underpins reduced cell proliferation. Transgenic Res. 25, 139-148.

34. Verleih M., Rebl, A., Köllner, B., Korytár, T., Anders, E., Wimmers, K., Goldammer, T., 2011. Comparative molecular characterization of the regucaclcin (RGN) gene in rainbow trout (Oncorhynchus mykiss) and maraena whitefish (Coregonus marena). Mol. Biol. Rep. 39, 4291-4300.

35. Wang, Z., Qu, Y., Zhuo, X., Li, J., Zou, J., Fan, L., 2019. Investigating the physiological responses of Pacific white shrimp Litopenaeus vannamei to acute cold-stress. Peer 7, e7381.

36. Yamaguchi, M., 2011. The transcriptional regulation of regulation of regucalcin gene expression. Mol. Cell Biochem. 346, 147-171.

37. Yamaguchi, M., 2013. The anti-apoptotic effect of regucalcin is mediated through multisignaling pathways. Apoptosis 18, 1145-1153.

\section{Tables}


Table 1

Primers used for qPCR

\begin{tabular}{|c|c|c|c|}
\hline Gene & Primer sequence $\left(5^{\prime}-3^{\prime}\right)$ & Amplicon size (bp) & Accession number \\
\hline Ccrgn & F: TGCTTTGATGGAGGAAGAGTG & 105 & MT218432 \\
\hline (E: 0.96) & R: TCCACCAAAACAGACGGATG & & \\
\hline Ccgapdh & F: TCCTGCACCACCAACTGC & 178 & XM_030783830 \\
\hline (E: 1.02) & R: GAGGCAGGGATGATGTTCTG & & \\
\hline Ccef1a & F: CCATTGTTCAGATGATTCCCG & 123 & XM_030788692 \\
\hline (E: 1.05) & R: CTTCTTGATGACACCAACAGC & & \\
\hline
\end{tabular}

Figures 
(A) ATGGCCTCTCCAAGA GTCGAATGTGTTGTG AAAGAGAGGAGTGAG ATTGGGGAGGGTCCA GTGTGGGAGGAAAAA 75

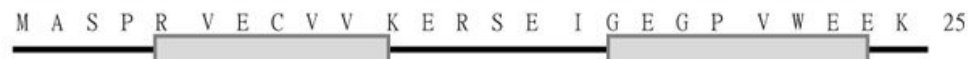
GAAAGCATTCTTCTG TATGTGGATGTCTGT GGTAGGCGCGTGAGC AGATGGAACTCGGCA ACCAATCAAATACAA 150

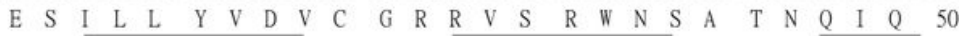
AGTCTTACCACAGAG AAATTTGTGGGATGC GTGGTTCCGCGGACG TCAGGAGGTTACATA GTATGCGAGGGGAGA 225 $\begin{array}{llllllllllllllllllllllllll}S & L & T & T & E & K & F & V & G & C & V & V & P & R & T & S & G & G & Y & I & V & C & E & G & R & 75\end{array}$

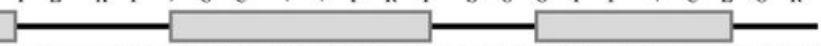

CGCTTCGCAGCATTA GACTGGGAGAAGCGA AGCCTGACCACCATT GCTGAGGTGGATAGC GACAAAGTCAACAAT 300 $\begin{array}{lllllllllllllllllllllllllll}R & F & A & A & L & D & \text { W } & E & K & R & S & L & T & T & I & A & E & V & D & S & D & K & V & N & N & 100\end{array}$ C CGCTTCAACGACGGC AAAGTCGATCCAGCT GGCAGGTTTTTCGCA GGCACAATGCTCCTA GACTGGGAGCAGGGA 375 $\begin{array}{llllllllllllllllllllllllll}R & F & N & D & G & K & \text { V } & D & P & A & G & R & F & F & A & G & T & M & L & L & D & W & E & Q & G & 125\end{array}$ P

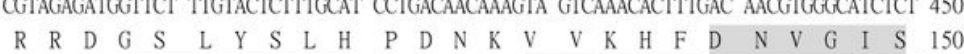
AACGGCCTGGACTGG TCTCTGGACCATCGA GTCTTCTACTACATC GACAGTTTGAACTAC ATGGTAGAGGCCTTT 525 $\begin{array}{llllllllllllllllllllllllll}\text { N } & G & \text { L } & \text { D } & \text { WI } & \text { S } & \text { L } & \text { D } & \text { H } & \text { R } & \text { V } & \text { F } & \text { Y } & \text { Y } & \text { I } & \text { D } & \text { S } & \text { L } & \text { N } & \text { Y } & \text { M } & \text { V } & \text { E } & \text { A } & \text { F } & 175\end{array}$ GATTACAACAAACAG ACCGGCAGCATCTCT AATCGCAGATCAGTA TACAAACTGGAGAAA GAAGAGGGTGTGCCT 600

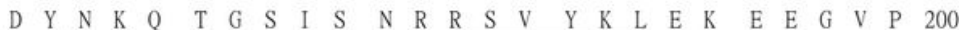
$\square$ GATGGTATGACCATT GATAATGAGGGAAAG CTCTGGGTTGCCTGC TTTGATGGAGGAAGA GTGCTACGTATTGAC 675 $\begin{array}{llllllllllllllllllllllllll}\text { D } & G & M & T & \text { I } & \text { D } & N & E & G & K & \text { L } & \text { W } & \text { V } & \text { A } & \text { C } & \text { F } & \text { D } & \text { G } & \text { G } & R & \text { V } & \text { L } & R & \text { I } & \text { D } & 225\end{array}$ CCACAGACAGGCGCT AGAGTTCAGACAGTA AAGCTGCCAGCTCAG AAGATCACATCOGTC TGTTTTGGTGGAAAA 750 $\begin{array}{llllllllllllllllllllllllll}P & Q & T & G & A & R & V & Q & T & V & K & L & P & A & Q & K & I & T & S & V & C & F & G & G & K & 250\end{array}$ P

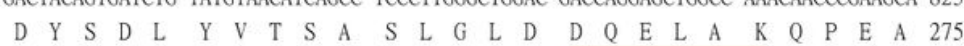
$\longrightarrow$ D GGATGCATATTTAGG GTTACAGGTCTCGGT GTGAAAGGACTTCCT CCTAATTCATTCGCT GGTTGAAATTTCAGT 900

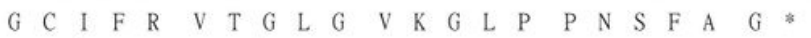
296

GAAATCTATTCCCGT TAGCTGTATCAGAAA AGGAATTGCGTTGAT CTAACACTTCATAAC AATGTCTCAATCACA 975 AACAAAACTATTCTT AAATTGTGTTAGAAA TCCGTTAAAGCATTG TTTGATTGGTTTCTC ATCAGCATGAAAAAC 1050 CTCAGCTTTGAAGGC AGTATGCGATGGATT ACAGCAATCTAGCTG TGCCAGACTGATCAT GCATCAGATTGATGG 1125 ACAATTTTTAAAACG GACCTAAAATGGATA ATGAAAAGGGTCTTT GGATAATCCTTTGGA ATTAAAAAGTGTACA 1200 TGAATGAAGCAGGAG TTGACTTTGACATGT TTTCATGTCTTGCAT TTTGCATTATGCTTC AGATAATTTCGTAAG 1275 GGATAATGTTCATCT GCAGATAATACTAAT ATACAGCAGTACCAG TAGAATAAGTATTAC CAATACAGGTAATAT 1350 TTAATACACGGTATG TCTTCCATTAGCTTT TGTGTAATGAGCTTG AAGTTTGGAAATTAA AAGTGAAAGCGTAAG 1425 GTTATTACAAATGAa GAACAACTGGaGGaA TGTAAAACATTTTTA GGTGAGCGCATTAAG AAAATAAAAAGGAAA 1500 AATGAAGAAAAAAAA AAAAAAAAA
(B)

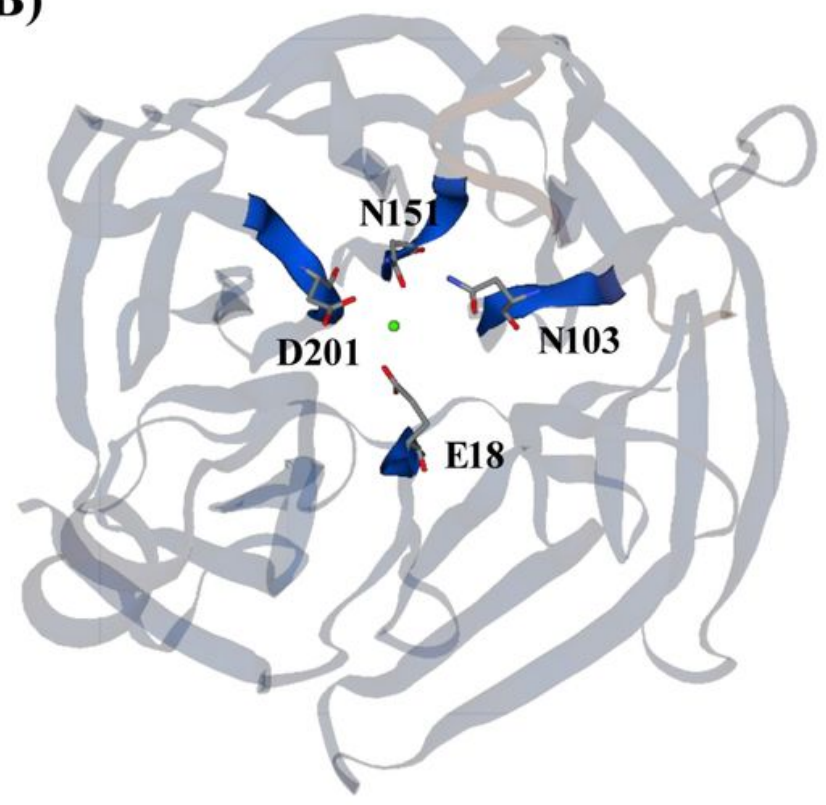

(C)

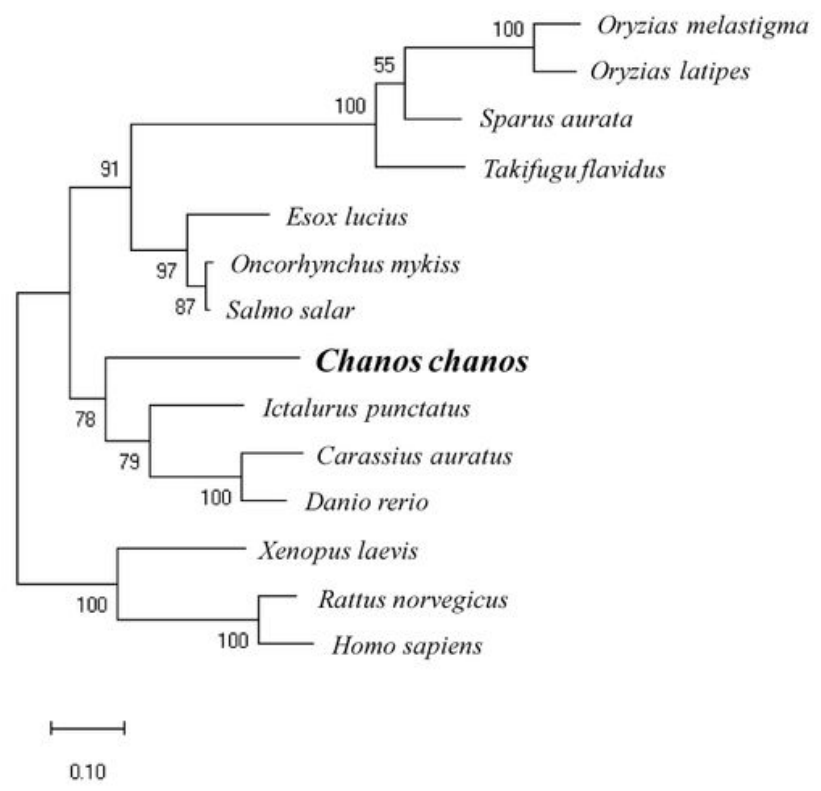

\section{Figure 1}

The characteristics of milkfish regucalcin sequence. (A) The full-length nucleotide sequence and predicted amino acid sequence of milkfish regucalcin. Gray box: $\beta$-strand; Red box: $a$-helix. (B) The predicted protein structure of milkfish regucalcin. Four residues (E18, N103, N151, and D201) are predicted to be the calcium binding residues (green spot). (C) Phylogenetic analysis of the amino acid sequence of milkfish regucalcin. The phylogenetic tree is construct using the Maximum likelihood method and the number indicates bootstrap values for 1000 replicates. The accession numbers of different species are listed in Supplementary Table 1. 


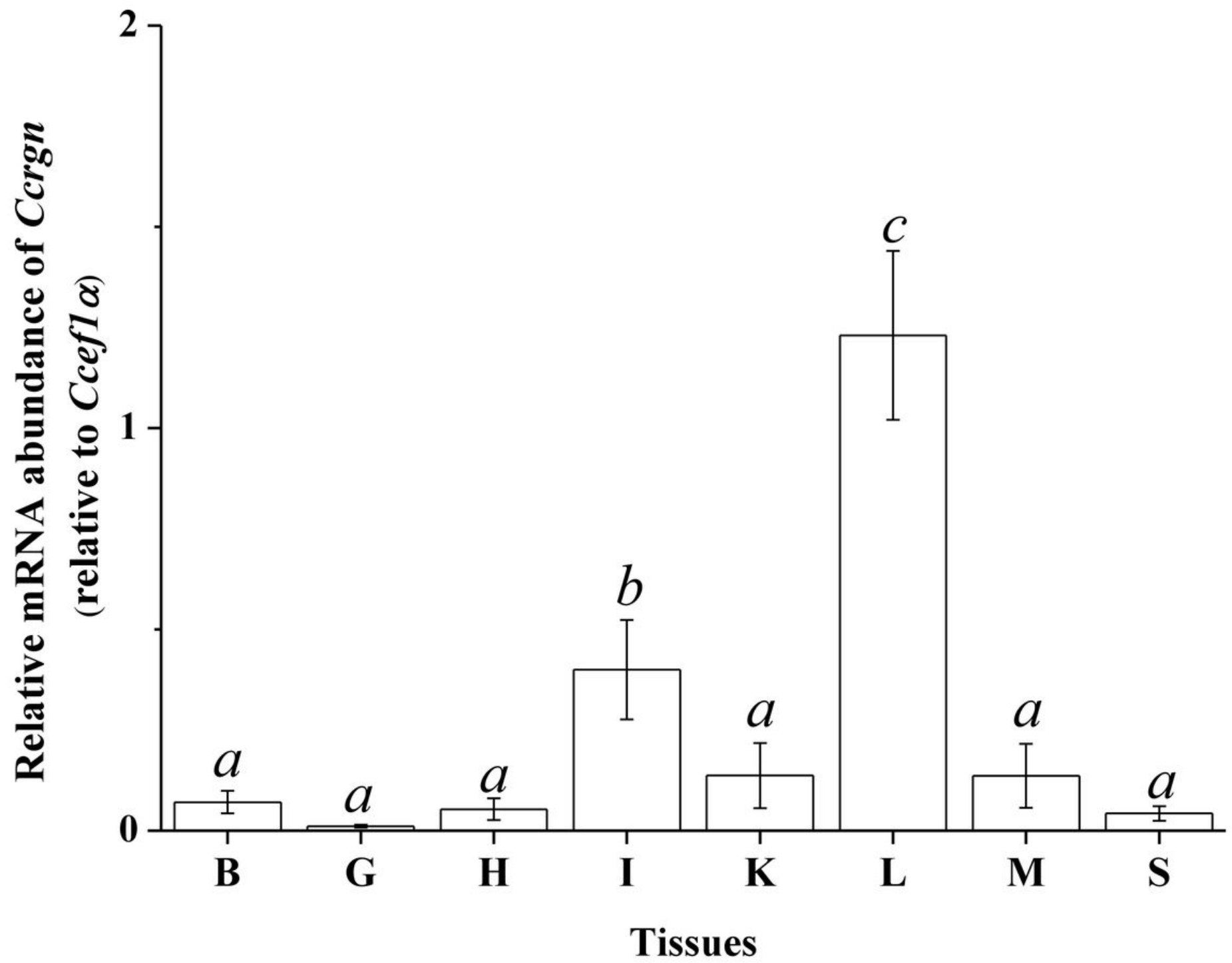

Figure 2

Tissue distribution of milkfish regucalcin gene (Ccrgn) analyzed by qPCR. Different letters indicate significant differences in Ccrgn expression among various tissues $(P<0.05$, one-way ANOVA, Tukey's test). Values are means $\pm S E M, n=3$. B, brain; $G$, gill; $H$, heart; I, Intestine; $K$, kidney; $L$, liver; $M$, muscle; $S$, spleen. 


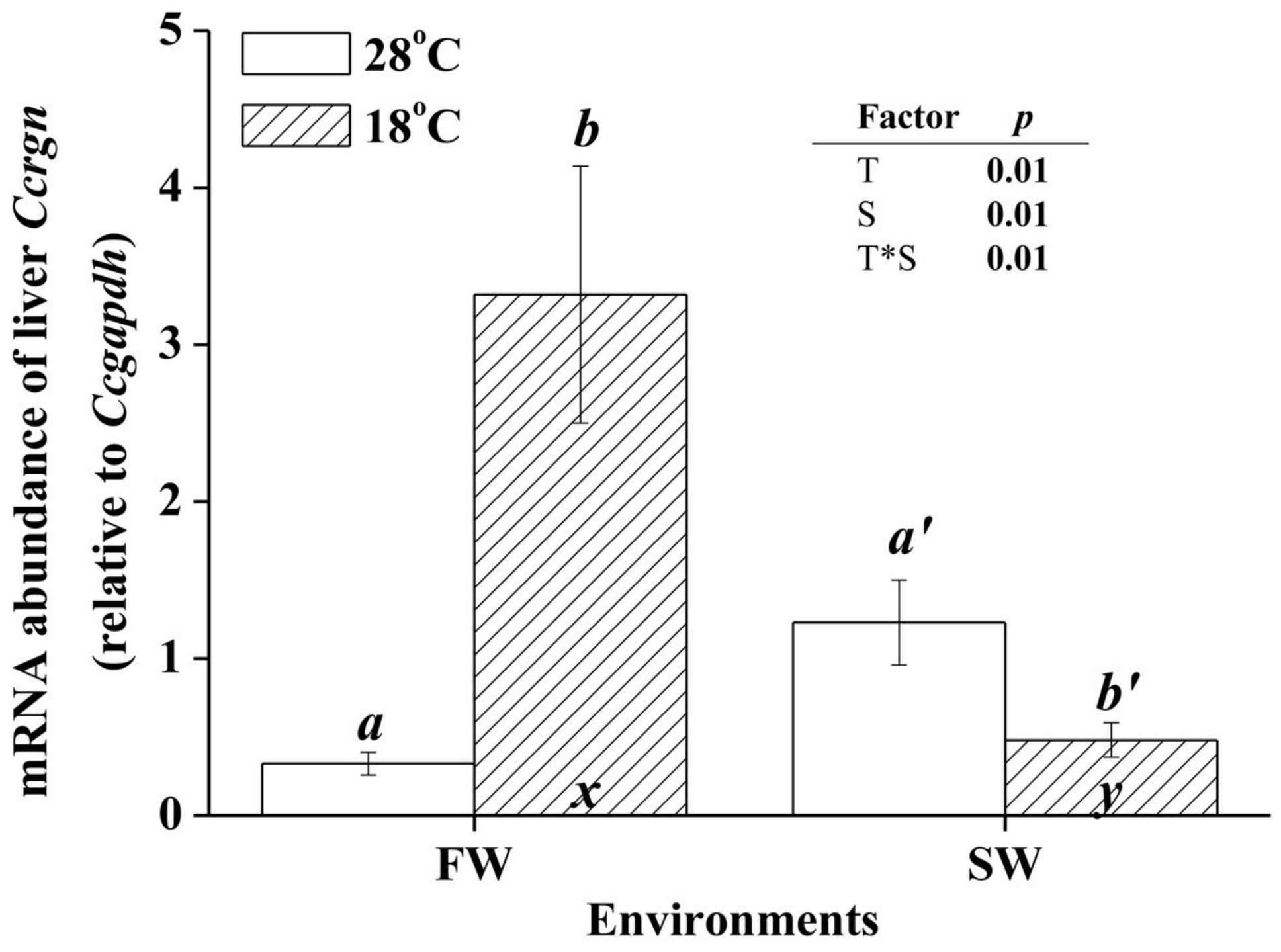

Figure 3

Expression of hepatic Ccrgn in fresh water (FW)- and seawater (SW)-acclimated milkfish under normal $\left(28^{\circ} \mathrm{C}\right)$ and hypothermal $\left(18^{\circ} \mathrm{C}\right)$ temperatures. Different letters in the FW milkfish $(\mathrm{a}, \mathrm{b})$ and SW milkfish $\left(a^{\prime}, b^{\prime}\right)$ indicate significant differences between the normal and low-temperature group. The $x$ and $y$ indicate significant differences between the FW and SW groups at $28^{\circ} \mathrm{C}$ or $18{ }^{\circ} \mathrm{C}(\mathrm{P}<0.05$, two-way ANOVA, Tukey's HSD pairwise comparison). Values are means $\pm S E M, n=6$. T, temperature; $S$, salinity. 


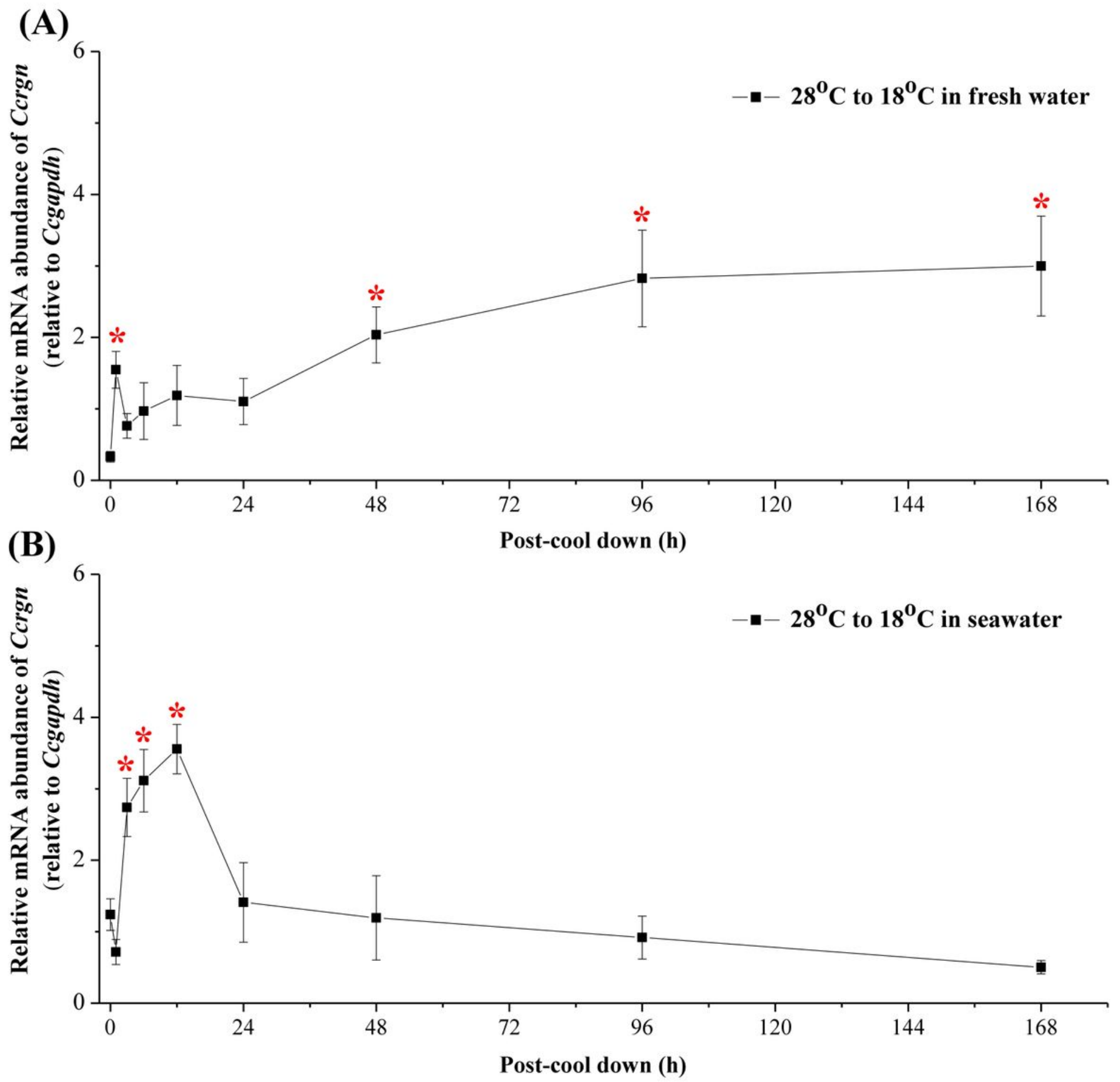

Figure 4

The time-course expression of hepatic Ccrgn in (A) fresh water-acclimated milkfish and (B) seawateracclimated milkfish upon hypothermal challenge. The asterisks $\left(^{*}\right)$ indicate significant differences between various time points and $0 \mathrm{~h}(\mathrm{P}<0.05$, one-way ANOVA, Dunnett's test). Values are means $\pm \mathrm{SEM}$, $\mathrm{n}=6$. 


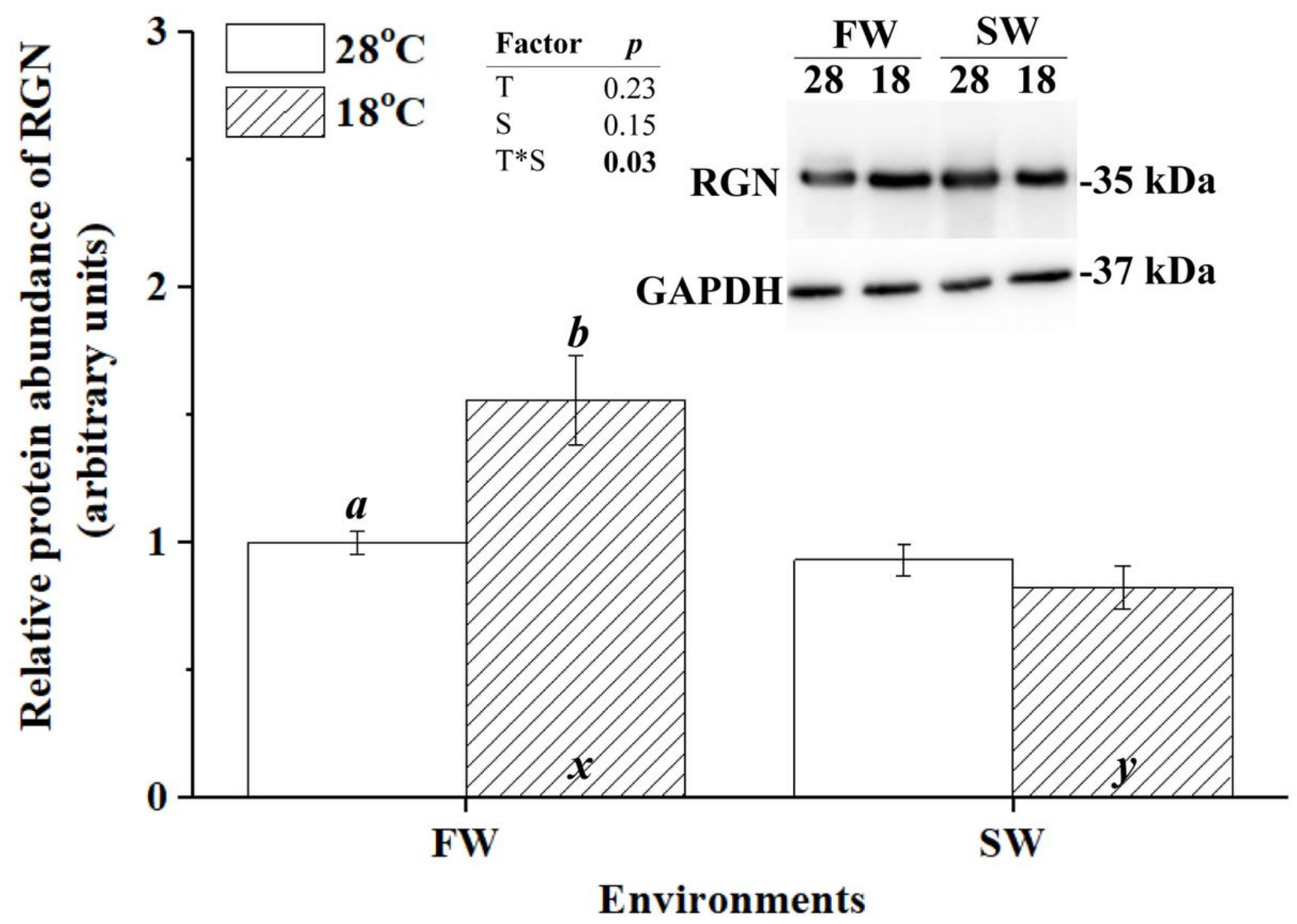

Figure 5

Relative protein abundance of hepatic regucalcin (RGN) in fresh water (FW)- and seawater (SW)acclimated milkfish at normal $\left(28^{\circ} \mathrm{C}\right)$ and hypothermal $\left(18^{\circ} \mathrm{C}\right)$ temperatures. The representative immunoblots of RGN showed a single immunoreactive band at $35 \mathrm{kDa}$. GAPDH as the loading control. Different letters $(a, b)$ indicate significant differences between the $28^{\circ} \mathrm{C}$ and $18{ }^{\circ} \mathrm{C}$ groups in $\mathrm{FW}$ or $\mathrm{SW}$. The $x$ and $y$ indicate significant differences between the FW and SW groups at $28^{\circ} \mathrm{C}$ or $18{ }^{\circ} \mathrm{C}(P<0.05$, two-way ANOVA, Tukey's HSD pairwise comparison). Values are means $\pm S E M, n=8$. T, temperature; $S$, salinity. 

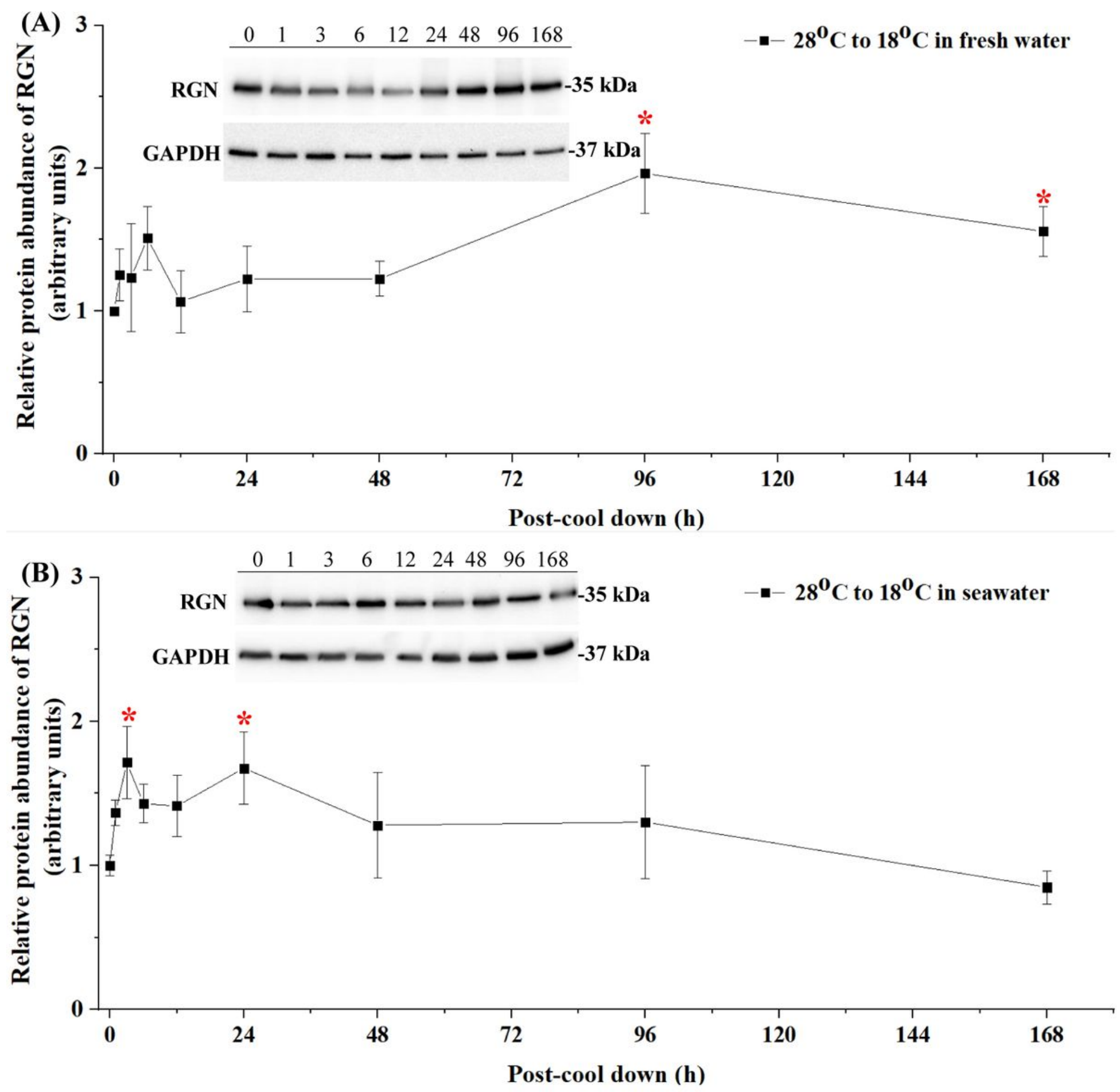

Figure 6

Relative time-course protein abundance of hepatic RGN in fresh water-acclimated milkfish (A) and seawater-acclimated milkfish (B) upon hypothermal challenge. The representative immunoblots of RGN shows a single immunoreactive band at $35 \mathrm{kDa}$. GAPDH as the loading control. The asterisks $\left({ }^{*}\right)$ indicated significant differences between various time points and $0 \mathrm{~h}(\mathrm{P}<0.05$, one-way ANOVA, Dunnett's test). Values are means $\pm S E M, n=6$. 


\section{Supplementary Files}

This is a list of supplementary files associated with this preprint. Click to download.

- SupplementaryTable1.docx 